\title{
Buchbesprechungen
}

Knochenmetastasen

Radiologische Diagnostik, Therapie und Nachsorge

K. Ríeden

Springer, Berlin, Heidelberg, New York 1988152 S., 49 Abb., 41 Tab., DM 98-ISBN 3-54019062-7

Das Thema «Knochenmetastasen» wird in zahlreichen spezia-lisierten Fachzeitschriften häufig aus der Sicht eines einzelnen Fachgebietes abgehandelt. Dieses Buch hat den unschätz-baren Vorteil, die klinisch radiologische und therapeutische Problematik in einem einzigen Band darzustellen. Hierbei greift die Autorin auf ein umfangreiches Literaturstudium zurück, welches auch neuere Veröffentlichungen berücksich-tigt. Diese Literaturdaten werden verglichen mit eigenen Er-gebnissen, die sich auf Untersuchungen an einem großen, statistisch aussagekräftigen Patientenkollektiv stützen. Der umfangreiche, straff gegliederte Text, bereichert durch Röntgendarstellungen, wird durch zahlreiche Tabellen und graphische Darstellungen sinnvoll ergänzt. Das Sachverzeich-nis erleichtert den schnellen Zugang zur Materie. Die Schwerpunkte der Darstellung orientieren sich an einem sinnvollen diagnostischen Vorgehen vor und nach Therapie knöcherner Metastasen. Der diagnostische Beitrag einzelner radiologischer Verfahren, orientiert an der klinischen Frage-stellung, wird im Hinblick auf ihre Aussagekraft, Effizienz. Rep Гoduzierbarkeit und wirtschaftliche Grundsätze analy-siert. Der Stellenwert der CT und MRT wird unterstrichen, wobei letzterer wegen noch begrenzter Verfügbarkeit, hohen ökonomischen Aufwandes und noch fehlender, vergleichender Studien genauer definiert werden muß. Das Werk, das auch in verlegerischer Hinsicht übersichtlich gestaltet ist, kann Radiologen, Onkologen und in angrenzen-den Disziplinen Tätigen vorbehaltlos empfohlen werden.

R. Westhaus, Starnberg

Nitrosamines

Toxicology and Microbiology

M.J. Hill (Hrsg.)

VCH Verlagsgesellschaft, Weinheim, Basel, Cambridge, New York 1988

169 S., 20 Abb., 25 Tab., DM 180,-

ISBN 3-527-26708-5

Das Buch ist sinnvoll gegliedert und präsentiert den Stoff verständlich. Abbildungen und Tabellen sind anschaulich und hinreichend groß. Der Literaturkatalog am Ende eines jeden Kapitels erlaubt ein schnelles Auffinden von Zitatstellen. Die Abkürzungsverzeichnisse am Ende der einzelnen Kapitel er-schweren das Nachschlagen; hier ware ein gemeinsames Abkürzungsverzeichnis (z.B. im Inneren des vorderen Buch-deckels) von Vorteil.

Das Buch bietet einen Überblick über Analyse, Chemie, Vorkommen und Toxikologie der Nitrosamine. Die verständ-liche Einfühmng in die Eigenschaften und Besonderheiten dieser Verbindungen hilft demjenigen, der sich erstmals mit dieser Materie auseinandersetzt. Aber auch dem Fachmann leistet der Band gute Dienste: als voUständiges und kurz gehaltenes 
Kompendium informiert es über den Stand der Forschungsarbeiten in den verschiedenen Fachrichtungen, Hinweise auf neuere Literatur erleichtern das Auffinden von Primärliteratur. Die Analyse von N-Nitrosoverbindungen unter Hinweis auf die ihr eigenen Probleme und Fehlerquellen beschrieben. Die Vor- und Nachteile verschiedener Verfahren, ihre Empfindlichkeit und Aussagefähigkeit werden deutlich. Die chemischen Eigenschaften und die Entstehung von Nitro-soverbindungen wird ebenso behandelt wie die Bedeutung dieser Vorgänge für Mensch und Umwelt. Ein besonderes Kapitel beschäftigt sich ausführlich mit der endogenen Entstehung von Nitrosaminen.

Das Kapitel über Vorkommen der Nitrosamine in Lebensmit-teln, Umwelt und Industrieprodukten stellt Daten zum Gehalt und Konzentrationen der Nitrosamine sowie deren Wechsel-wirkungen zusammen. Die berufsbedingte Exposition ist ebenfalls berücksichtigt. Die Ergebnisse toxikologischer Forschung an Nitrosaminen werden über den gesamten Bereich der in-vivo und in-vitro-Versuche hinweg diskutiert. Dem Nitrosamin-bedingten Krebs beim Menschen ist ein besonderes Kapitel gewidmet, das Tumorinzidenzen und -lokalisationen in Beziehung zur Nitro-samin-Exposition setzt.

Anhand all dieser Daten wird eine Risikoabschätzung vor-genommen und die Perspektiven zukünftiger Forschungen besprochen.

Insgesamt ist das Buch eine wertvolle Hilfe in der Diskussion um die Bedeutung und Gefährlichkeit der Nitrosamine, außer-dem ein gut konzipiertes Werk, das den Einstieg in die Thema-tik und die Orientierung im breiten Literaturangebot erleichtert.

\section{U.Mohr, Hannover}

$$
\text { í.íti. ív }
$$

ix täglich*

TARGOCIO

[tEicopla $\mathrm{i}$ i $]$

Buchbesprechungen

Buchbesprechungen

223

Targocid $\AA$ 100/Targocid $\beta$ 200/Targocid $® 400$

Zusan $\cap$ mensetzung:

11njektionsflascheTargocid 100/200/400 enthält $114 \mathrm{mg} /$

$224 \mathrm{mg} / 424 \mathrm{mg}$ Trockensubstanz e $\mathrm{tsprechend} 100 \mathrm{mg} /$

$200 \mathrm{mg} / 400 \mathrm{mg}$ Teicoplanin; 1 Ampulle enthält 1,8 ml/

3,2 ml/3,2 ml Lösungsmittel (Wasserfür Injektionszwecke).

Anwendungsgebiete:

Durch grampositive Erreger verursachte mittelschwere und schwere Infektionen des Herzens (z. B. Endokarditis), der Knochen und Gelenke (z.B. Osteomyelitis), der Atemwege, der Haut und des Weichteilgewebes, der Niere und der ableitenden Harnwege und des Magen-Darm-Traktes; Sepsis, Septikämie. Zur perioperativen Prophylaxe bei erhöhter Gefährdung des Patienten durch grampositive Infektionen. Gegenüber gramnegativen Bakterien ist Teicoplanin nichtwirksam. Teicoplanin istauch bei Erregern (vor allem bei Staphylokokken) wirksam, die gegenüber Cephalosporinen, Oxacillin oder Methicillin resistent sind.

Gegeranzeigen:

Bekannte Uberempfindlichkeit gegenüber dem Wirkstoff, Schwangerschaft und Stillzeit, Kinder unter 3 Jahren. Nicht 
in den Liquorraum applizieren.

Hinweis:

Bei Patienten mit eingeschränkter Nierenfunktion soilte die

Therapie sorgfältig überwacht werde $\cap$. Im Falle einer

Behandlungsdauer von mehr als drei Wochen werden

regelmäßige Kontrollen des Serumspiegels sowie der

Nieren-, Leber- und Hörfunktion empfohlen (besondersfür

Patienten mit eingeschränkter Nierenfunktion).

Nebenwirkunqen:

Gelegentlich Uberempfindlichkeit gegenüber dem Wirkstoff mit Exanthemen, Erythemen,

Juckreiz oder Fieber, in Einzelfälien Bronchospasmen oder anaphylaktische Reak-tionen. Als

lokale Reaktion gelegentlich an der Injektions-stelle Schmerzen, vereinzelt Phlebitis oder

Abszedierung. In einigen Fallen passagerer Anstieg derTransaminasen und/oderderalkalischen

Phosphatase. In Einzelfälien Anstieg des Serumkreatinins. Selten Eosinophilie, Thrombozytopenie oder Leukopenie. Im Magen-Darm-Bereich selten Úbelkeit und Erbrechen, vereinzelt Kopfschmerz oder Schwindel. Die in Einzelfälien beobachteten Nebenwirkungen wie leichter Hörverlust, Tinnitus oder eine vesti-buläre Störung traten überwiegend nur bei Patienten auf, die zusätzlich zur Therapie mit Teicoplanin potentiell ototoxisch wirkende Arzneistoffe, wie z. B. Aminoglykoside, erhielten.

Wechselwirkungen mitanderen Mitteln: In klinischen Studien sind bisher keine

Wechselwirkungen mit anderen Arzneimitteln beobachtet worden. Dennoch wird eine sorgfältige Überwachung der Nieren- und Hörfunktion empfohlen, wenn Teicoplanin in Kombination mit Substanzen verabreicht wird, von denen bekannt ist, daß sie die Nieren- und Hörfunktion beeinträchtigen können. Stand:Januar1989

Merrell Dow Pharma GmbH 6090 Rüsselsheim

Im Mitvertrieb von:

Lederle Arzneimittel GmbH \& Co

8190 Wolf ratshausen

UICC

International Union against Cancer TNM-Klassifikation maligner Tumoren

Herausgeber und Übersetzer: P. Hermanek, O. Scheibe,

B. Spiessl, G. Wagner

Springer, Berlin 1987

4., vollständig neu überarbeitete Neuauflage

XVIII, 213 S., broschiert, DM 22,-

ISBN 3-540-17602-0

Die 4. Auflage zur Klassifikation maligner Tumoren nach dem TNM-System liegt nunmehr vor. Sie wurde gründlich über-arbeitet und wiederum in handlichem Format erstellt, was bei der Anwendung in der Klinik sehr hilfreich ist. Die Bedeutung einer standardisierten Klassifikation von Tü-morerkrankungen für die Klinik ist unbestritten. Und so ist es auch hervorzuheben, daß wichtige Erkenntnisse über Progno-sefaktoren, die den Therapieentscheid beeinflussen, in die Klassifikation Eingang gefunden haben. Einzelne Kapitel wur-den intensiv überarbeitet, besonders die über Tumoren des Magen-Darm-Traktes. Die Einteilung der Tumoren anderer Organsysteme wurde an Klassifikationssysteme adaptiert, die im klinischen Alltag sich bereits länger bewährt hatten. Ungeachtet vieler positiver Entwicklungen des TNM-Systems gegenüber früheren Auflagen bleibt die klinische Relevanz einiger Neuerungen allgemeinen Inhalts und zu 
speziellen Organtumoren off en. So wird durch die weitergehende Diffe-renzierung des histopathologischen Gradings die Vergleich-barkeit von Statistiken und Studien, die auf der vorausgegan-genen Auflage basierten, mit neueren Statistiken problema-tisch. Bei der Beschreibung des C-Faktors («Diagnosesiche-rung») verwundert die beschriebene Gleichrangigkeit eines Befundes, der z.B. durch bildgebende Verfahren, ohne Biop-sie und Zytologie erhoben wurde, mit diagnostischen Maßnah-men, die durch endoskopische Untersuchungen immerhin zur Biopsie und zytologischen Untersuchung führten. Und schließlich wird die neu eingeführte R-Klassifikation (Resi-dualtumor) zunächst wohl nur für die R0- und R2-Kategorie Bedeutung gewinnen, aber ohne Zweifel noch eine weitere Differenzierung erfahren müssen.

Bei einigen Organtumoren werden bereits bekannte klinisch relevante Erkenntnisse Berücksichtigung finden müssen. So ist aus meiner Sicht insbesondere bei den Hodentumoren das TNM-System noch unbrauchbar.

Ungeachtet der insgesamt wenigen Unzulänglichkeiten, ist die Bedeutung der 4. Auflage des TNM-Systems groß und für die klinische Arbeit aller Ärzte, die sich mit der Diagnostik und Therapie maligner Erkrankungen beschäftigen, unverzicht-bar.

P.T., München 